\title{
A Study on Outputting the Shape of Carpus using Medical Image and 3D Printer
}

\author{
Hyeong-Gyun Kim', Joon -Koo Choi ${ }^{*}$, Gha-Jung Kim', Dong-Hee Hon' and Ki-Moo Lim² \\ 'Department of Radiology Science, Far East University, Chungbuk, Korea; \\ jung7818@hanmail.net, rslaser@hanmail.net,gjms1225@hanmail.net, hansound2@hanmail.net \\ 2Department of Medical IT Convergence Engineering, Kumoh National Institute of Technology, \\ Gumi, Gyeongbuk, Korea; kmlim@kumoh.ac.kr
}

\begin{abstract}
Background/Objectives: This research is an experimental research to output the DICOM image of carpus through 3D printer and to confirm the identity of 3D medical image and the shape. An experimental analysis was conducted as follows: First, the size on the 3D medical image and the length of the shape output through 3D printer were measured and compared using Digital Vernier Calipers; second, the anatomical forms and shapes before and after an experimental study were evaluated based on a survey of evaluators composed of 10 medical imaging experts and the results were reflected. Methods/Statistical Analysis: The purpose of an experiment in this research is to contribute to improving learning effects by manufacturing a phantom for medical learning about carpus and to establishing a clinical diagnosis and treatment plan. Carpus was used as the object of an experiment. The reason is that since carpus has the very irregular and smallest bones in human body, it has advantages in comparing the precision of a manufactured shape and improves educational understanding in medical learning. Findings: According to the research results, the length of the longitudinal plane in carpus used for the experiment was $120 \mathrm{~mm}$ equally in the medical image and the shape output through 3D printer. As the results of comparing the form and shape of carpus output through 3D printer with DICOM image of carpus, radiologists represented 16\% for Good and 84\% for Very Good and radiological technicians represented 2\% for Fair, 20\% for Good and 78\% for Very Good. Improvements: In conclusion, if the material equivalent to human body can be developed and its shape can be output through 3D printer, the learning effects through a phantom is expected to be greatly improved.
\end{abstract}

Keywords: Carpus, DICOM Image, Phantom, Shape, 3D Printer

\section{Introduction}

3D printing technology is a type of technology to manufacture the digital data acquired through threedimensional scan or three-dimensional modeling by the additive manufacturing method. In the 1980s, Stereo Lithography (SLA) and Fused Deposition Modeling (FDM) were devised. Since then, derivative technologies have been developed and utilized not only at the level of prototype manufacturing but also as functional parts in various industrial fields ${ }^{1,2}$. The utilization of this 3D printing technology has recently been expanded into the manufacturing of customized prosthetic legs, dental prosthetics, artificial vessels, bio chips other than tissue engineering scaffolds. In particular, 3D printing technology in medical fields utilizing the function of visualizing digital data can be efficiently utilized for advance planning for surgical operation and the tools for actual practice ${ }^{3}$. Siamese twin separation surgery performed in the U.S. UCLA Hospital in 2002 is the first typical case of the application of visualization ${ }^{4}$.

The separation surgery depending on existing medical imaging took about 100 hours because of the complex structure of cerebrovascular and nervous systems,

*Author for correspondence 
whereas an operation was successfully finished in 22 hours by printing out MRI images via 3D printer, establishing a surgical plan and performing preliminary practices. In complex surgical operation, surgical simulation application based on this CT and MRI medical imaging technology has been utilized in preventing medical malpractices and increasing surgical efficiency.

This research intends to apply 3D printing technology using the visualization of medical images to this research, to output the 3D printing shape of carpus composed of several bones, which are the smallest and complex bones among human body joints and to confirm whether or not the shape is equivalent to the form. This can improve the quality of education by using a phantom equivalent to human body for medical education and training and clinically contribute greatly to establishing a diagnosis and treatment plan for patients.

\section{Materials and Methods}

\subsection{Methods}

DICOM (Digital Imaging and Communication in Medicine) image of carpus acquired using medical image was image-processed into three-dimensional data and converted into STL (Stereo Lithography) file that could be perceived by $3 \mathrm{D}$ printer. Photo Sensitive Liquid Polymer in a liquid state was irradiated and laminated by layer and a technology for building three-dimensional shape structure was applied ${ }^{5}$. To compare the precision of an output shape, the length of an output shape to medical image was measured using a measuring instrument for engineering. To compare the identity with the form of shape surface, a survey of five radiologists and five radiological technicians was conducted and the results were derived [Figure 1] [Figure 2].

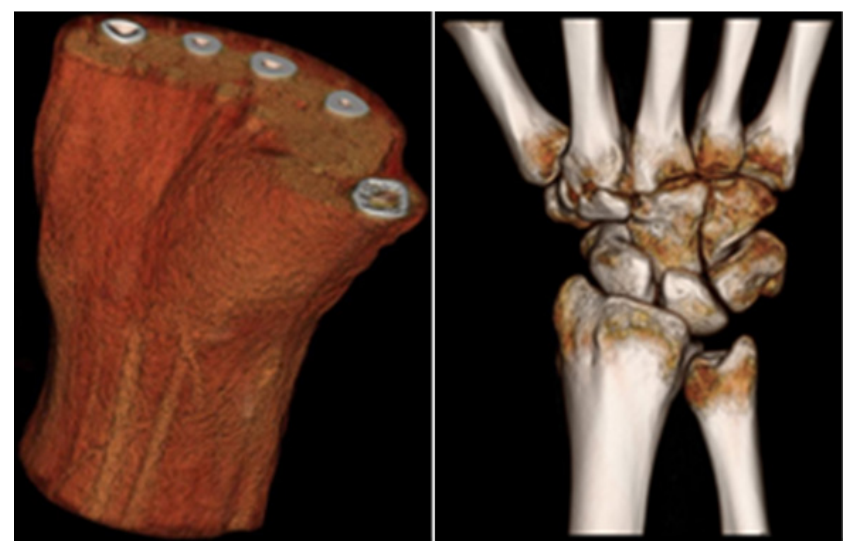

Figure 1. 3D image of carpus by DICOM file.

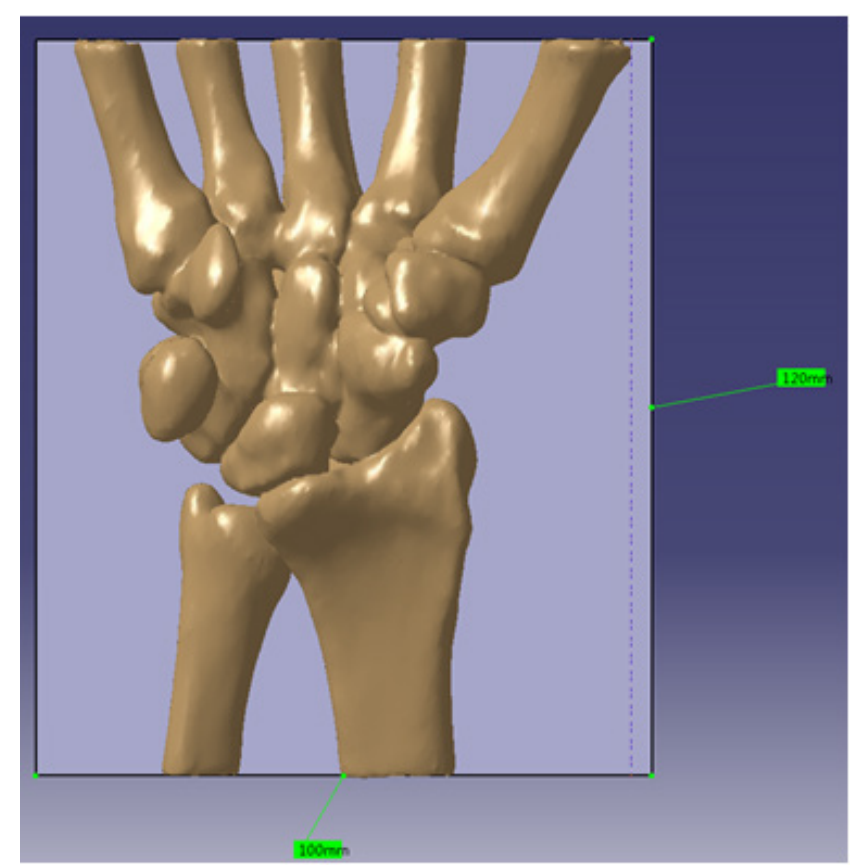

Figure 2. 3D image of carpus converted into STL file.

\subsection{Materials}

The $0.5 \mathrm{~mm}$ Volume data of 640 Multi Slice Computed Tomography (640 MSCT, Aquilion ONE, Toshiba, Japan) was used in acquiring the image of carpus using medical image. Objet30 Pro-Desktop 3D Printer (Stratasys, USA) was used in outputting the shape via 3D printer [Table 1]. Photo Sensitive Liquid Polymer in a liquid state was used as material for 3D printer. To embody 3D image for medical image, STL (Stereo Lithography) conversion file was created using Terarecon (USA) 3D program. The size of the output shape of carpus was measured using Digital Vernier Calipers (Mitutoyo, Japan).

Table 1. 3D printing used in outputting the image of carpus

\begin{tabular}{ll}
\hline Specification & Size \\
\hline Output bed & $300 \mathrm{~mm}$ x $200 \mathrm{~mm} \times 150 \mathrm{~mm}$ \\
Nozzle diameter & $\mathrm{mm}$ \\
Layer thickness & $28 \mu \mathrm{m}(16 \mu \mathrm{m}$ and more $)$ \\
\hline
\end{tabular}

\section{Results}

\subsection{Measurement of the Size of Shape}

To measure the size of an output shape, the length of the shape of carpus output after setting the $120 \mathrm{~mm} \mathrm{X} 100$ $\mathrm{mm}$ original-sized bed using 3D image program was directly measured using Digital Vernier Calipers [Figure 3]. The length of the longitudinal plane of carpal bones 
was equally $120 \mathrm{~mm}$ and a transverse plane was 94.25 $\mathrm{mm}$, which represented the length measured actually within the size of the bed. This means that the size of a shape output by $3 \mathrm{D}$ printer in this experiment was the same as the length represented by medical image.

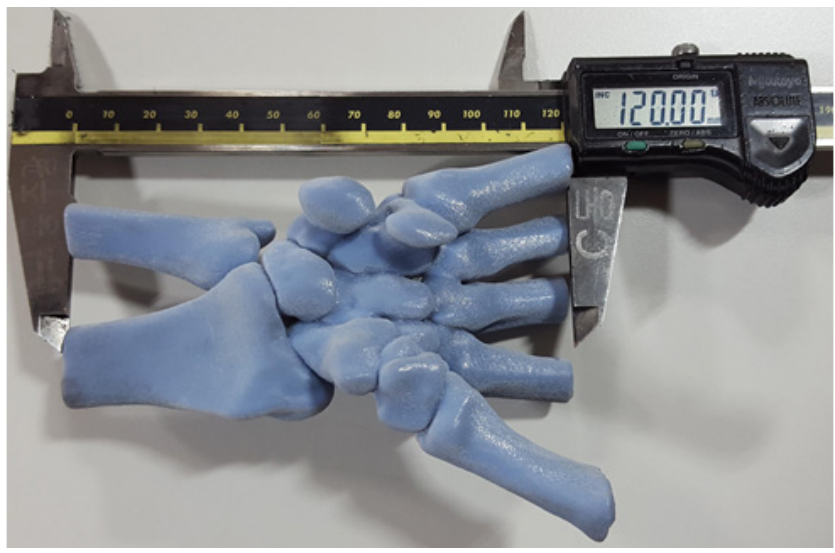

Figure 3. The length of the longitudinal plane of carpal bone shape output by $3 \mathrm{D}$ printer.

\subsection{Evaluation of Anatomical Structure of the Shape and Articular Surface of Carpus}

It was compared and evaluated whether or not the shape of carpus output by 3D printing was appropriate as the form seen on medical image. Each bone was described in terms of human anatomical features.
Looking into the posterior and anterior of the output shape, five radiologists and five radiological technicians responded to presented questions [Figure 4]. A questionnaire was prepared that, among five responses established in terms of anatomical features of eight carpal bones, one response could be selected and evaluated. Eight among ten items included questions about whether or not anatomical boundary is made clear against the forms of each carpal bones and the neighboring articular surface. Two items included questions about whether or not the array of metacarpal bone and forearm bone and the differentiation of the overall articular surface are made clear. In the evaluation of overall sub-items, radiologists represented 16\% for Good and 84\% for Very Good and radiological technicians represented $2 \%$ for Mediocre, $20 \%$ for Good and 78\% for Very Good. In particular, all the two groups of radiologists and radiological technicians represented the projecting feature of medial hamate bone as Very Good. However, in the evaluation as to whether or not the articular surface of carpus is clearly differentiated, radiologists represented $80 \%$ for Good and $20 \%$ for Very Good. Radiological technicians represented $20 \%$ for Mediocre and $80 \%$ for Good. These are relatively low compared with other items. Five items to radiologists and four items to radiological technicians were evaluated as Very Good by all the evaluators. No item was evaluated as bad and the below by either of two groups [Table 2] [Table 3].

Table 2. Questionnaire item for image specialists to compare output shapes ${ }^{18}$

\begin{tabular}{|c|c|c|c|c|}
\hline \multirow[t]{2}{*}{ Item } & \multicolumn{4}{|c|}{ Radiologists (Radiological technicians) $\mathrm{N}=5(5)$} \\
\hline & Very bad Bad & Mediocre & Good & Very Good \\
\hline $\begin{array}{l}\text { Is scaphoid the largest in body-side array and ship-shaped and jointed } \\
\text { with trapezium, trapezoid, capitate, lunate and radius? }\end{array}$ & & & $2(1)$ & $3(4)$ \\
\hline $\begin{array}{l}\text { Is lunate half moon-shaped and jointed with scaphoid, capitate, hamate, } \\
\text { triquetrum and radius? }\end{array}$ & & & & $5(5)$ \\
\hline $\begin{array}{l}\text { Is triquetrum pyramid-shaped and jointed with capitate and pisiform on } \\
\text { palm? }\end{array}$ & & & 2(1) & $3(4)$ \\
\hline Is pisiform bean-shaped? & & & (1) & $5(4)$ \\
\hline $\begin{array}{l}\text { Is trapezium saddle-shaped and jointed with the 1st and 2nd metacarpus, } \\
\text { trapezoid and scaphoid? }\end{array}$ & & & & $5(5)$ \\
\hline $\begin{array}{l}\text { Is trapezoid jointed with 2nd metacarpus, trapezium, capitate and } \\
\text { scaphoid? }\end{array}$ & & & (2) & $5(3)$ \\
\hline $\begin{array}{l}\text { Is capitate jointed with the } 2^{\text {nd }} \text {, the } 3^{\text {rd }} \text { and the } 4^{\text {th }} \text { metacarpus, trapezoid, } \\
\text { scaphoid, lunate and hamate? }\end{array}$ & & & & $5(5)$ \\
\hline Is hamate wedge-shaped and does it have coronoid process on palm? & & & & $5(5)$ \\
\hline $\begin{array}{l}\text { Is each articular surface of carpal bones clearly differentiated from each } \\
\text { other? }\end{array}$ & & (1) & $4(4)$ & 1 \\
\hline $\begin{array}{l}\text { Are metacarpus, carpus, radius and ulna in proper three-dimensional } \\
\text { arrangement? }\end{array}$ & & & (1) & $5(4)$ \\
\hline
\end{tabular}


Table 3. Results of two groups' evaluation of the overall items

\begin{tabular}{llcccc} 
& \multicolumn{1}{c}{} & (unit: \%) \\
\hline Classification & Very bad & Bad & Mediocre & Good & Very Good \\
\hline Radiologists & 0 & 0 & 0 & 16 & 84 \\
Radiological technicians & 0 & 0 & 2 & 20 & 78 \\
\hline
\end{tabular}
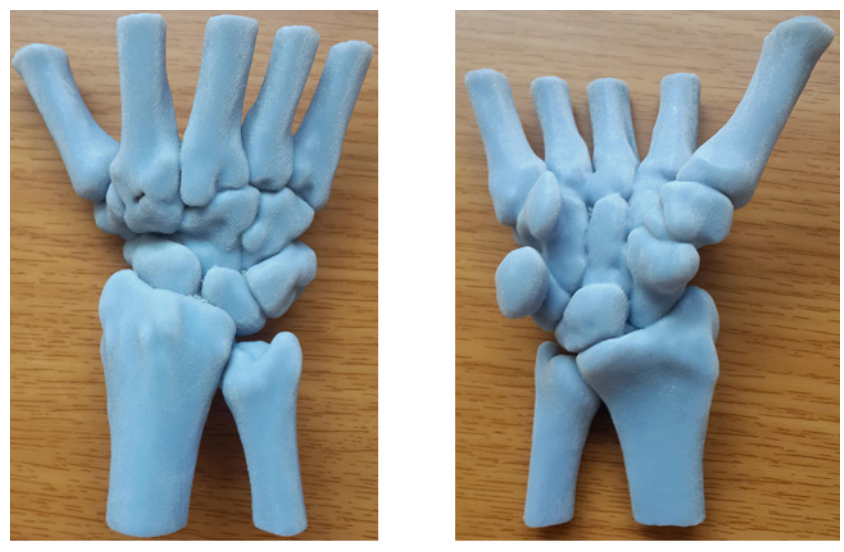

(a) Posterior

Figure 4. Posterior and anterior of carpal bone shape output by $3 \mathrm{D}$ printer.

\section{Discussion}

The CT equipment used in this research is medical diagnosis equipment used for different purposes ${ }^{6}$ such as diagnosis of various diseases, the selection of the scope of mass excision before surgical operation and the evaluation of surgical site after operation. 3D printer using the method of laminating polymer (or resin) in a liquid state on layer-by-layer basis and manufacturing a shape $^{7}$ was selected to output the shape of DICOM data acquired by medical equipment. With the development of $3 \mathrm{D}$ printer, the clinical usefulness of the model of broken bone for surgical operation was proved by numerous previous studies ${ }^{8-11}$. Conducted a study on applying the model of real shaped bone to surgical planning, the study of prosthetic techniques and the pre-education of surgical operation has been done using CT data ${ }^{12}$.

Carpal bones are very small and irregular in form and different in size. Also their several joints are adjoined to each other. So, carpal bones are advantageous in confirming the precision of $3 \mathrm{D}$ printer. The effects of learning about different forms are high in manufacturing a phantom. That is the reason why carpus was selected as the experiment target. However, there was a limitation that

image specialists check the precision indirectly because of the absence of tools to measure the precision. Besides, there was also a limitation that a clear classification could not be made when the floating articular surface of human body was represented in a fixed shape. Eight shapes of carpal bones were output in a complete form without being separated. The three-dimensional shape structures of metacarpal bones and palm bones were difficult to visually differentiate from the shapes seen on medical image.

In particular, the three-dimensional shape structures of scaphoid, lunate, triquetrum, pisiform, trapezium, trapezoid, capitates and hamate were properly represented consistent with each characteristic of the eight bones in convex and concave surfaces ${ }^{13}$. Since a human hand plays an important role in the human body, many researchers and engineers are trying hard to develop a robot hand with the function similar to the function of a human hand $^{14.15}$. Further, the materialization of bone structures outside the human body and the resultant expansion of understanding are expected to benefit assistive technology area, exoskeleton robot area and rehabilitation area ${ }^{16-18}$.

\section{Conclusions}

Based on a study on outputting the images of carpus represented using diagnostic medical imaging in the same shapes through $3 \mathrm{D}$ printer, the following conclusion was derived. This research identified that the size and shape of carpus seen on medical image was manufactured in the same form and real shape. As the results of measurement using Digital Vernier Calipers, it was found that the length was exactly consistent. Also, according to the results of an investigation of the anatomical form and structure of carpus by medical image specialists, all items were evaluated as Good and the above. This will increase an expectation of phantom learning in medical education and training by manufacturing a real shape of carpus of human body and furthermore, contribute greatly to establishing a clinical diagnosis and treatment plan. 


\section{References}

1. Ahn DG, Yang DY. Principle of rapid prototyping and its trends. Korean Journal of Precision Engineering. 2005; 22(10):7-16.

2. Wohler T. Wohler's Associates Inc. Wohlers Report; 2013.

3. Park SH, Park JH, Lee HJ, Lee NK. Current status of biomedical applications using 3D printing technology. J Korean Soc Precis Eng. 2014; 31(12):1067-76.

4. Turkcadcam. Rapid prototyping helps separate conjoined twins. 2014. Available from: http://www.turkcadcam.net/ rapor/ Otoinsa/uyg-medikal-conjoined-twins.html

5. Kang BH, Sin SR, Jin SE, Im MR, Lee SJ, Kim JS. Experiment of solidifying photo sensitive polymer by using UV LED. Korean Society for Precision Engineering Spring Conference 2008 proceedings; 2008 Nov.

6. Kim DH, Ko SJ, Kang SS, Kim JH, Choi SY, Kim C. Evaluation of image quality and dose with the change of $\mathrm{kVp}$ and BMI in the Liver CT. Journal of the Korean Contents Association. 2013; 13(6):331-8.

7. Kwak KH, Park SW. Global 3D printer industry technology trends analysis. J of the KSME. 2013; 53(10):58-64.

8. Honiball JR. The application of $3 \mathrm{D}$ printing in reconstructive surgery. Dissertation presented in fulfillment of the requirements for the degree MscIng in Industrial Engineering at the University of Stellenbosch; South Africa. 2010.

9. Frame M, Huntley JS. Rapid prototyping in orthopaedic surgery: A User's guide. The Scientific World Journal; 2012 May.

10. Oh WK, Lim KS, Lee TS. Additive manufacturing of pa- tient-specific femur via 3D printer sing Computed Tomography Images. Journal of the Korean Society of Radiology. 2013; 7(5):359-64.

11. Goto M, Katsuki T, Noguchi H, Hino N. Surgical simulation for reconstruction of mandibular bone defects using photocurable plastic skull models: Report of three cases. 1997 Jul; 55(7):772-80.

12. Seoung YH. 3-Dimensional printing for mesh types of short arm cast by using computed tomography. Journal of the Korean Contents Association. 2015; 15(1):308-15.

13. Kim HY, Choi JK, Kim GJ, Hong DH, Cho HJ. Study on reproduction of shape by $3 \mathrm{D}$ printer using DICOM image of carpus. The 5th International Conference on Convergence Technology in 2015 Korea Convergence Society; 2015.

14. Krechetov IV, Skvortsov AA. Approach to the study of kinematics and modeling grip of 22 DOF anthropomorphic gripping manipulator. Indian Journal of Science and Technology. 2015 Dec; 8(S10):1-9.

15. Deimel R, Brock O. A novel type of compliant and underactuated robotic hand for dexterous grasping. The International Journal of Robotics Research; 2015 Aug. p. 1-9.

16. Palanivendhan $M$, Wadhawan $M$, Selvagandhi R. Upper-limb shape memory alloy orthosis for restoration or improvement of basic hand functions. Indian Journal of Science and Technology. 2015 Oct; 8(27):1-4.

17. Lo HS, Xie SQ. Exoskeleton robots for upper-limb rehabilitation: State of the art and future prospects. Medical Engineering and Physics. 2012 Apr; 34(3):261-8.

18. Lampignano J, Kenneth L. Bontrager textbook of radiographic positioning and clinical diagnosis. 8th ed. 2014. 